\title{
DERECHO INTERNACIONAL Y POLÍTICA DE ADAPTACIÓN AL CAMBIO CLIMÁTICO EN EL REINO UNIDO*
}

Gabriel Araya Ahumada**

\section{Resumen}

Este artículo describe la influencia que ha ejercido el derecho internacional desde las orientaciones provenientes del régimen internacional del cambio climático y la Unión Europea, en la gobernanza actual de la adaptación al cambio climático en el Reino Unido, especialmente en relación con la política del desarrollo sustentable.

Palabras clave: cambio climático, adaptación, desarrollo sustentable, planes de adaptación, programas de adaptación.

\section{INTERNATIONAL LAW AND THE CLIMATE CHANGE ADAPTATION POLICY IN THE UNITED KINGDOM}

\begin{abstract}
This article describes in what ways international environmental law, the international regime of climate change and the European Union have influenced current climate change adaptation governance in the United Kingdom, especially in relation with the sustainable development policy.
\end{abstract}

* Artículo preparado en el marco de las investigaciones realizadas por el área legal del Centro de Ciencia del Clima y la Resiliencia (CR)2, Centro de Excelencia Fondap-Conicyt 15110009, del cual el autor es asistente de investigación, y al cual expresa sus agradecimientos. Quisiera agradecer de manera particular a la doctora Marie-Claire Cordonier y al doctor Markus Gehring, miembros del Lauterpacht Centre for International Law, de la Universidad de Cambridge, por su cálida acogida y asistencia en la investigación.

** Abogado. Candidato a Magíster en Derecho, Universidad de Chile. Santiago de Chile (Chile). [garaya@ ug.uchile.cl]

Recibido: 17 de marzo de 2016 / Modificado: 5 de agosto de 2016 / Aceptado: 1 de septiembre de 2016

Para citar este artículo

Araya Ahumada, G. (2016). Derecho internacional y política de adaptación al cambio climático en el Reino Unido. OPERA, 19, pp. 11-34.

DOI: http://dx.doi.org/10.18601/16578651.n19.03 
Key words: Climate change, adaptation, sustainable development, adaptation plans, adaptation programs.

\section{INTRODUCCIÓN}

El Reino Unido forma parte del Anexo uno de la Convención Marco de las Naciones Unidas sobre Cambio Climático (en adelante CMNUCC o Convención), lo que en la práctica se traduce en mayores obligaciones dentro de dicho régimen internacional ${ }^{1}$. Este país, además, es miembro de la Unión Europea (UE) y de la Organización para la Cooperación y el Desarrollo Económico (OCDE), organizaciones internacionales que han prestado particular atención a la problemática del cambio climático, elaborando lineamientos y regulaciones que, con diferentes grados de vinculatoriedad, determinan la acción de los Estados en el ámbito doméstico.

Asimismo, el régimen del cambio climático en el Reino Unido posee una particularidad regulatoria que lo distingue a nivel interna- cional: fue el primer país en tener una ley de cambio climático en el mundo, la Climate Change Act del año 2008. Esta ley, destacada en el campo de la mitigación ${ }^{2}$, comienza a exhibir sus primeros frutos en adaptación, tras la publicación del primer Programa Nacional de Adaptación (NAP, por sus siglas en inglés) para el Reino Unido, en 2013.

Sin embargo, el NAP no es únicamente resultado de la aplicación de la Climate Change Act. Por el contrario, se nutrió de los esfuerzos regulatorios y de política pública expresados en otros ámbitos de la política ambiental del Reino Unido, particularmente en relación con el desarrollo sustentable, lo cual es además coherente con los lineamientos internacionales en la materia.

Otro aspecto que conviene tener presente en este análisis tiene relación con los desafíos propios que plantea la adaptación al cambio climático como problema de política pública, los cuales, por cierto, exceden el ámbito ambiental $^{3}$, e impactan de manera diferente a las diferentes poblaciones y territorios afectados; a la vez que mantiene todavía altos grados de

\footnotetext{
1 "El régimen internacional del cambio climático está conformado por dos instrumentos obligatorios: la Convención marco de las Naciones Unidas sobre cambio climático [...] y el "Protocolo de Kioto" [...]. A ellos se agregan las múltiples decisiones de las Partes, que si bien en principio no son obligatorias, su estatus jurídico es bastante discutido y en la práctica este deberá calificarse según la decisión de que se trate, y sin perjuicio de que muchas veces en la práctica operan de facto como tales" (Urrutia, 2010). Para una reflexión sobre la naturaleza jurídica de los documentos emanados de los diversos órganos del régimen climático véase Vihma (2012).

2 Para Reid (2012), las modificaciones realizadas sobre los sectores energético y tributario, junto a la aplicación de otras medidas aisladas, no contenidas en la Climate Change Act, habrían contribuido al éxito en el campo de la mitigación.

3 "El fenómeno no es solamente un problema ambiental. En realidad, sus consecuencias negativas pueden ser tan disímiles y complejas como para afectar en distinta medida a prácticamente todas las actividades relevantes para el género humano: la salud global, el acceso a las fuentes de agua, la seguridad alimentaria, el uso de la energía y la protección de la biodiversidad, e incluso la propia existencia de algunos países" (Urrutia, 2010).
} 
incertidumbre y escasa evidencia científica para sustentar la toma de decisiones. Finalmente, tampoco existe una única metodología para monitorear los resultados de las medidas de adaptación aplicadas.

La literatura en el campo del diseño de medidas de adaptación ha intentado sistematizar los desafíos anteriormente descritos a través de las siguientes interrogantes: ¿Cómo integrar mejor las políticas de adaptación a nivel horizontal a través de todos los sectores? ¿Cómo integrar mejor la adaptación a través de los diferentes niveles jurisdiccionales? ¿Cómo integrar el conocimiento relevante en las decisiones de adaptación? Y ¿ ¿ómo involucrar a un amplio rango de actores no estatales que serán afectados por el cambio climático pero que a menudo carecen de las capacidades necesarias para adaptarse? (Bauer, Feichtinger y Steurer, 2012).

Las respuestas a estas preguntas dependen de la elección soberana de cada Estado, sin embargo, la decisión debería considerar los lineamientos de carácter general establecidos en la Convención, precisados cada vez con mayor detalle por el trabajo de la Conferencia de las Partes (COP); y para el caso de los países miembros de la Unión Europea, por el régimen comunitario, a través de los Libros Blanco y Verde, y la Estrategia de Adaptación. En consecuencia, para estudiar y comprender la política de adaptación en el Reino Unido es necesario analizar previamente las orientaciones y directrices que se originan a nivel internacional.

\section{CONTEXTO INTERNACIONAL Y COMUNITARIO DE LA ADAPTACIÓN AL CAMBIO CLIMÁTICO}

\section{Tratamiento de la adaptación en el régimen internacional del cambio climático}

La adaptación no está definida de manera expresa dentro de la Convención. Al respecto, Schipper comenta que durante el proceso de negociación de la CMNUCC, Australia y Nueva Zelanda presentaron como concepto de adaptación "toda actividad deliberada e intencional tomada de manera anticipada o en respuesta a los efectos del rápido cambio climático" (Schipper, 2006), el cual no prosperó en el texto definitivo, quedando en manos de la doctrina dicha tarea.

En efecto, Biagini identifica tres conceptos de adaptación ${ }^{4}$ (Biagini, Bierbaum, Stults,

\footnotetext{
4 "Ajuste de los sistemas humanos o naturales frente a entornos nuevos o cambiantes. La adaptación al cambio climático se refiere a los ajustes en sistemas humanos o naturales como respuesta a estímulos climáticos proyectados o reales, o sus efectos, que pueden moderar el daño o aprovechar sus aspectos beneficiosos" (Panel Intergubernamental de Cambio Climático), "un proceso mediante el cual estrategias para moderar, enfrentar y tomar ventaja de las consecuencias de los eventos climáticos (en oposición con solo el cambio climático antropogénico) son mejoradas, desarrolladas e implementadas" (Programa de las Naciones Unidas para el Desarrollo) y finalmente, "ajuste en los sistemas ecológicos, sociales y económicos en respuesta a los estímulos climáticos actuales o esperados y sus efectos o impactos. Se refiere a los cambios en procesos, prácticas y estructuras para moderar los daños potenciales o para beneficiarse de las oportunidades asociadas con el cambio climático" (sitio web de la Convención) (Biagini, Bierbaum, Stults, Dobardzic y McNeeley, 2014).
} 
Dobardzic y McNeeley, 2014), los cuales difieren entre sí en el énfasis de la adaptación como un efecto, un ajuste de los sistemas naturales o humanos, o un proceso tendiente a un resultado en particular. En cambio, todas estas definiciones comparten como elemento la preparación de la sociedad para los efectos positivos y negativos del cambio climático (Least Developed Countries Expert Group, 2012).

Los autores, además, distinguen varios artículos repartidos a lo largo de la CMNUCC que tratan implícitamente la adaptación. Entre ellos se mencionan los artículos 2, 4.1, literales B, E y F, 4.4, 4.8 y el artículo 4.9. Este último es de suma relevancia, pues tipifica aquellas circunstancias nacionales que reflejarían los diferentes factores de vulnerabilidad de los Estados.

Los avances en adaptación al cambio climático durante los primeros ańos de la aplicación de la CMNUCC fueron mucho más lentos que aquellos logrados en mitigación. Esto se explica dado que la mitigación fue la principal preocupación de los Estados partes, tal como quedara de manifiesto en el mandato de Berlín de 19965. Mientras que para algunos autores lo anterior se justificaba en la escasa información en relación con los efectos del cambio climático y la vulnerabilidad (UNFCCC,
2006), para otros dicha situación obedeció principalmente a que la adaptación "fue vista como 'una idea inaceptable, incluso políticamente incorrecta' (Burton, 1994, 11), porque enfocarse en la adaptación era percibido como una excusa para evitar tareas de reducción de emisiones de gases de efecto invernadero más difíciles. La preocupación sobre la adaptación podía ser moralmente peligrosa" (Schlosberg, 2013). Finalmente, otros autores afirman que el lento desarrollo de la adaptación se debió a la inexistencia de disposiciones explicitas al interior de la Convención (Schipper, 2006).

Cualquiera sea la explicación, es posible dividir el proceso de adaptación al interior de la Convención estableciendo el cisma en 2001, año en que fue publicado el III Informe del Panel Intergubernamental de Cambio Climático ${ }^{6}$ (PICC). Dicho reporte alertó sobre la existencia de ciertos impactos en el planeta inevitables en el corto plazo (esto es, perceptibles dentro de las próximas dos décadas) elevando la necesidad de lidiar con estos impactos a través de la adaptación.

De esta suerte, la adaptación es tratada en la primera Conferencia de las Partes (1995) de manera tangencial al abordar otras temáticas, tales como: los requisitos de las Comunicaciones Nacionales; las funciones que se asignan al Órgano Subsidiario de Asesoramiento

5 La postergación de la adaptación en favor de la mitigación también es evidente en el régimen comunitario. Al respecto véase Mehling (2009).

6 Lo cual, por lo demás, es coincidente con otra división propuesta por Huq y Toulmin (2006), quienes fraccionan el proceso general del cambio climático -es decir, considerando mitigación y adaptación- en tres etapas: desde 1988 hasta 2001, desde dicha fecha hasta 2007 y de 2007 hasta el presente, estableciendo como hitos el Primer, Tercer y Cuarto Reporte del PICC. 
Científico y Tecnológico (osact) ${ }^{7}$ (reunir, difundir y evaluar las informaciones, relativas a la adaptación); o los requisitos para acceder a financiamiento a través del mecanismo financiero (CMNUCC, 1995).

En las decisiones de las partes no hay evidencia de cambios significativos en los siguientes cinco años. Sin embargo, hay en esta etapa dos acontecimientos que contribuirán a establecer las bases para el posterior cambio de actitud de la comunidad internacional en relación con la adaptación. En primer lugar, se inició un proceso encaminado a identificar medidas de adaptación para atender a las necesidades de los países en desarrollo, de conformidad con los párrafos 8 y 9 del artículo 4 de la CMNUCC ${ }^{8}$.

La segunda excepción está contenida en el artículo 12.8 del Protocolo de Kioto, que destina a la adaptación una parte de los fondos obtenidos a través del Mecanismo de Desarrollo Limpio. La importancia de la creación de este fondo no radica tanto en la recaudación obtenida, sino en el hecho de que para asignar los fondos se hizo necesaria la elaboración de estándares que permitieran discriminar entre los diversos proyectos provenientes de los países demandantes (Least Developed Countries Expert Group, 2012), abriendo de ese modo un espacio de reflexión en torno a cuáles serían las prioridades en adaptación que convenía financiar.

La importancia relativa de la adaptación comienza a aumentar tras la publicación del $3^{\circ}$ Informe del PICC, el que, como se dijo, llama a los Estados a prepararse ante los impactos climáticos inevitables. Esta nueva perspectiva fue condicionada además por la constatación de que los países más vulnerables, en relación con el artículo 4.9 de la cMNUCC, eran también los más pobres y menos preparados para llevar adelante procesos de adaptación; mientras que, por el contrario, muchos países desarrollados ya habían elaborado sus programas nacionales de adaptación, en cumplimiento del artículo 4.1 (b) de la cMnUCC. A partir de este momento, las Partes advierten la urgencia de apoyar la adaptación de aquellos países menos preparados para hacerse cargo de esta problemática.

Como resultado de lo anterior, la Conferencia de las Partes estableció el Programa de Trabajo para los Países Menos Adelantados (PMA) en los Acuerdos de Marruecos del año 2001. Este programa está dirigido principalmente a orientar y apoyar a los países que por sí solos no han avanzado en la adaptación al cambio climático, mediante: 1) la introducción de directrices para la elaboración de Programas de Acción Nacionales de Adaptación

La CMNUCC contempla en sus artículos $9^{\circ}$ y $10^{\circ}$ la creación de dos órganos subsidiarios de carácter técnico: el Órgano Subsidiario de Asesoramiento Científico y Tecnológico (оSACT), y el Órgano Subsidiario de Ejecución (OSE).

8 Aplicación de los párrafos 8 y 9 del artículo 4 de la CMnucc. A través del mismo se allanó el camino para la adopción del Programa de Trabajo en el Marco del Plan de Buenos Aires, proceso que proveería a su vez los antecedentes necesarios para la posterior preparación de los Acuerdos de Marruecos (cMnUcc, 1998). 
(NAPA, por sus siglas en inglés) destinados a los PMA, para que estos últimos identifiquen y reporten sus necesidades de adaptación, y hagan propuestas sobre cómo enfrentarlas; 2) el establecimiento de un Grupo de Expertos para los Países Menos Adelantados (GEPMA) que asesore a estos en la preparación de los NAPA y facilite la difusión de información ${ }^{9} ; 3$ ) la creación de un fondo para los países menos adelantados, destinado a preparar y financiar la implementación de los NAPA; y finalmente, 4) un programa de trabajo independiente para los PMA (CMNUCC, 2001).

La profundización de este proceso ocurriría recién en 2004, año en que las Partes acordaron el denominado Programa de Trabajo de Buenos Aires sobre las Medidas de Adaptación y de Respuesta ${ }^{10}$. Si bien Verschuuren (2012) califica este programa como un "intento débil para tratar la adaptación, especialmente en los países en desarrollo", lo cierto es que este Programa hace extensiva la invitación a participar del proceso a los países en desarrollo, promoviendo la realización de diversas actividades destinadas a mejorar la calidad en las metodologías para la recolección y el análisis de las informaciones proporcionadas en relación con la adaptación.

El Programa de Trabajo de Buenos Aires es absorbido, dos años más tarde, por el Programa de Nairobi. Este último, con una visión más ambiciosa del proceso que se venía llevando a cabo, contiene dos objetivos principales: 1) mejorar la comprensión y evaluación de las partes acerca de los impactos, las vulnerabilidades y la adaptación al cambio climático; y 2) ayudar a las partes a tomar decisiones mejor informadas, sobre bases científicas, técnicas y socioeconómicas, tomando en cuenta la variabilidad actual y futura del cambio climático.

La progresiva certidumbre ${ }^{11}$ con la cual cada informe del PICC va sosteniendo la existencia del cambio climático y su origen an-

9 El objetivo del Grupo de Expertos de los Países Menos Adelantados es asesorar sobre la preparación y la estrategia de aplicación de programas nacionales de adaptación (PNA) que puedan atender a las necesidades inmediatas y apremiantes de los países menos adelantados (PMA) en materia de adaptación (CMNUCC, 2001).

10 Las medidas de respuesta, comprendidas como "cualquier posible consecuencia adversa que las políticas y medidas de mitigación adoptadas por las Partes del anexo II puedan tener en los países en desarrollo y la manera de minimizar esas consecuencias", son tratadas a partir de 2001 junto al análisis de las medidas de adaptación. Asimismo, en esta Conferencia se le encomienda al оsACT la elaboración de un programa quinquenal de trabajo que sería aprobado por la cop 11 y cuyo objetivo es: ayudar a todas las partes, en particular a los países en desarrollo, incluidos los países menos adelantados y los pequeńos Estados insulares en desarrollo, a mejorar su comprensión y evaluación de los impactos, la vulnerabilidad y la adaptación, y adoptar decisiones informadas sobre actividades y medidas prácticas de adaptación para hacer frente al cambio climático (CMNUCC, 2001).

11 Viñuales lo resume de la siguiente manera: "En su primer informe, la conclusión del ipcC era muy cauta: 'la detección inequívoca del aumento del efecto invernadero a partir de observaciones no parece probable por una década o más'. En su segundo informe, la conclusión permanece cauta, pero puede observarse claramente un cambio de acento: 'el balance de la evidencia [...] sugiere una influencia humana discernible sobre el clima global'. El tercer informe prosigue en la misma dirección: 'existe nueva y sólida evidencia de que la mayor parte del calentamiento observado en los últimos 50 ańos es atribuible a actividades humanas'. Finalmente, el cuarto informe menciona de 
trópico es categóricamente reafirmada en el Cuarto Reporte del PICC, publicado en el año 2007. La respuesta de la Conferencia de las Partes, contenida en el Plan de Acción de Bali, es una hoja de ruta con el ambicioso objetivo de dar cabal cumplimiento a toda la Convención. En relación con la adaptación, este plan se traduce en la promoción de cuatro ámbitos: 1) el apoyo a la implementación de acciones urgentes; 2) la consideración de las estrategias y los medios de reducción de desastres y medios para enfrentar los denominados loss and damage; 3) la accesibilidad y calidad del apoyo financiero y técnico; y 4) mejorar el desarrollo y la transferencia de tecnología.

Pero será recién en la cop de Cancún, que la adaptación -como seńalara Cristina Figueres- "hasta aquel entonces la Cenicienta de la Convención, encuentre su zapato de cristal" (Econoticias.com, 2011). En esta COP, las partes afirmaron por primera vez que la adaptación tenía el mismo nivel de prioridad que la mitigación, estableciéndose el Marco de Adaptación de Cancún (CAF, por sus siglas en inglés), con el objetivo de intensificar la labor relativa a la adaptación.
El caf está estructurado sobre la base de cinco elementos: 1) implementación; 2) apoyo internacional, que comprende cooperación financiera "predecible adicional y de largo plazo"; 3) la creación del Comité de Adaptación, para coordinar la implementación del CAF y de las medidas de adaptación (CMNUCC, 2010); 4) el respeto a los principios contenidos en la Convención, a los cuales se añade además una perspectiva de género y transparencia; $y$ 5) el compromiso con stakeholders para involucrar al sector privado y a la sociedad en todos sus niveles.

La implementación es el elemento de mayor importancia del Marco de Cancún, pues promueve la ejecución de acciones concretas, la mejora en las estrategias de reducción de riesgos de desastres relacionados con el cambio climático, y el establecimiento de un proceso tendiente a la elaboración de planes nacionales de adaptación (CMNUCC, 2010).

Las cop de Durban, Doha, Varsovia y Lima (CMNUCC, 2014) profundizan el desarrollo del CAF, especialmente en relación con la definición del rol y las tareas asignadas al Comité de Adaptación ${ }^{12}$ y la discusión en tor-

modo mucho más decidido que 'el calentamiento del sistema climático es inequívoco' y que 'es altamente probable [más de $90 \%$ de probabilidad] que la mayor parte del aumento observado en la temperatura global promedio desde mediados del siglo xx se deba al aumento observado en la concentración de gases de efecto invernadero de origen antropógeno" (Viñuales, 2009).

12 La Conferencia de las Partes afirmó que el Comité de Adaptación sería el órgano consultivo general de la cop sobre la adaptación y estableció las cinco funciones que le corresponderían: a) ofrecer orientación y apoyo de carácter técnico a las partes; b) fortalecer, consolidar y potenciar el intercambio de la información en todos los niveles; c) promover las sinergias y reforzar la colaboración con organizaciones, centros y redes nacionales, regionales e internacionales; d) ofrecer información y recomendaciones, basadas en las buenas prácticas de adaptación; y e) examinar la información comunicada por las partes acerca de la vigilancia y el examen de las medidas de adaptación (CMNUCC, 2011). 
no a las directivas para la elaboración de planes nacionales de adaptación (CMNUCC, 2012).

Recapitulando, la preocupación por la adaptación al cambio climático estuvo determinada en gran medida por la evidencia científica emanada del PICC, respecto a los impactos que el cambio climático supondría para los Estados. El creciente pesimismo de dichos reportes motivó a las partes a pasar de una actitud más bien indiferente a una actitud activa, caracterizada al principio por la lenta generación e intercambio de información relativa a la adaptación, para posteriormente dar lugar a la promoción de una planificación efectiva para la adaptación, la cual estuvo dedicada principal, pero no exclusivamente, a la colaboración con los países más necesitados.

\section{Programas de adaptación y planes de adaptación: orientaciones desde la Convención}

1. Programas nacionales de adaptación. Para Verschuuren, el mayor desafío de la adaptación tiene que ver con la complejidad y necesidad de adaptar los sistemas normativos ${ }^{13}$, cuestión que pudo ser mejor enfrentada por los países desarrollados (Biesbroek et al., 2010) al disponer de capacidades y recursos suficientes. En cambio, la situación de los países en desarrollo, especialmente los PMA, haría imprescindible la asistencia y el apoyo internacional, tanto en la formulación, como en la implementación y el financiamiento de las medidas de adaptación. A raíz de lo anterior, la Conferencia de las Partes adoptó, en los denominados Acuerdos de Marruecos del año 2001, las Directrices para la elaboración de los Programas Nacionales de Adaptación ${ }^{14}$ (NAPA), invitando a los PMA a utilizarlas de conformidad con sus circunstancias nacionales.

Los NAPA son concebidos como un cauce simplificado y directo para comunicar información sobre las necesidades de adaptación urgentes e inmediatas de los PMA. Son, en otras palabras, un mecanismo de información sobre las necesidades de adaptación de los PMA, a través de las cuales pueden acceder a financiamiento internacional (CMNUCC, 2001).

En este contexto parece natural que las directrices establezcan lineamientos generales que deben satisfacer los NAPA: deben estar orientados a la acción, contener actividades priorizadas y ser de fácil comprensión. Adicionalmente, se estableció un conjunto de "elementos indicativos", que corresponden a factores o materias que deben guiar "entre otros" la elaboración de los NAPA, por ejemplo,

13 En sus palabras: "El mayor problema, sin embargo, es la dificultad inherente de adaptar la política y la ley. Mientras que las medidas de mitigación pueden ser primariamente implementadas en un campo de política (el derecho ambiental), las medidas de adaptación deben ser implementadas a través de un amplio rango de políticas" (Verschuuren, 2012).

14 Directrices para la preparación de los programas nacionales de adaptación (CMNUCC, 2001). 
la multidisciplinariedad, la igualdad de género, el desarrollo sostenible, etc.

Existen además algunos lineamientos de carácter formal, dirigidos al proceso de elaboración de los NAPA, dentro de los cuales cabe destacar la promoción de la participación ciudadana desde la formulación. Finalmente, otros aspectos formales contenidos en las directrices hacen referencia a la estructura que debe seguir el documento, lo cual facilita la comparación entre diferentes NAPA.

2. Planes nacionales de adaptación. Como se señaló, la cop 16 estableció un proceso para que los PMA pudieran formular y ejecutar planes nacionales de adaptación (PNAD) invitando a las partes que son países en desarrollo a emplear las modalidades formuladas $-\mathrm{y}$ por formular- para respaldar los PNAD al realizar labor de planificación (CMNUCC, 2010).

La siguiente COp aprobó las directrices iniciales para la formulación de PNAD, cuyos objetivos son: a) reducir la vulnerabilidad frente a los efectos del cambio climático mediante el fomento de la capacidad de adaptación y de la resiliencia; y b) facilitar la integración de la adaptación al cambio climático, de manera coherente, en las políticas, las actividades y los programas pertinentes nuevos y ya existentes (CMNUCC, 2011). Por último, las formulaciones destacan que la planificación para la adaptación es un proceso continuo, progresivo e itera- tivo, que debe ser realizado con arreglo a las circunstancias nacionales y de acuerdo con las demás políticas existentes a fin de estar coordinado con los objetivos, planes, políticas y programas nacionales de desarrollo sostenible.

Como se aprecia, la diferencia entre los programas nacionales de adaptación y los planes nacionales de adaptación no es solo una cuestión terminológica sino también de objetivos. Mientras que en los programas el verbo rector es "comunicar", en el caso de los planes hay un doble objetivo: reducir la vulnerabilidad e integrar la adaptación en las demás políticas nacionales, lo cual da cuenta de una mirada más amplia sobre el problema (Least Developed Countries Expert Group, 2012). Apoyando estos nuevos objetivos, la Conferencia de las Partes le encarga al GEPMA preparar las directrices técnicas para los PNAD. Este grupo cumplió el mandato ${ }^{15}$, señalando que el proceso: 1) no debe ser prescriptivo, clarificando que las directrices tienen como propósito asistir a los PMA; 2) debe mejorar la coherencia de la planificación de la adaptación y el desarrollo a fin de evitar duplicidades; 3 ) debe facilitar la conducción del proceso por cada país; 4) está diseñado para que los países puedan revisar, monitorear y actualizar periódicamente sus PNAD; 5) identificar de manera permanente vacíos en capacidad y adaptación (Least Developed Countries Expert Group, 2012).

15 OsE. Informe de la 23a Reunión del Grupo de Expertos para los países menos adelantados (Ejecucción, 2013). 
Por otra parte, cabe destacar que entre las directrices iniciales y las directrices técnicas hay una relación de causa-efecto. Efectivamente, mientras el Anexo correspondiente establece cuatro aspectos por considerar: 1) establecimiento de las bases y determinación de las carencias, 2) elementos preparatorios, 3) estrategias de aplicación, 4) supervisión, evaluación y presentación de informes, las directrices técnicas se extienden sobre cada uno de estos, señalando, por ejemplo, iniciativas en que cada Parte se puede involucrar o las preguntas específicas que cada uno está llamado a responder.

La bondad de este sistema es que conjuga una decisión que, sin ser obligatoria, es el resultado consensuado de la negociación $y$, por tanto, de cierta relevancia jurídica para las partes, con un documento de carácter operativo, aunque eminente e indiscutiblemente voluntario. Es decir, respeta la libertad propia de cada Estado a la vez que proporciona herramientas para facilitar el cumplimiento de los objetivos.

\section{Tratamiento de la adaptación en el marco de la Unión Europea (UE)}

La política de cambio climático al interior de la Unión Europea ha seguido un camino similar al del régimen internacional estable- cido por la CMNUCC. En virtud de lo anterior, mientras que la mitigación ha sido objeto de amplio desarrollo ${ }^{16}$, la adaptación ha seguido una marcha más lenta, tanto respecto de las medidas adoptadas por cada país a nivel nacional, como en el ámbito supranacional, en relación con las medidas comunitarias.

En este contexto, la uE ha ejercido su influencia, alentado a los Estados miembros a desarrollar medidas de adaptación, pero con una mirada global de la problemática, entendiendo que los impactos pueden generar consecuencias positivas y negativas mucho más allá del espacio geográfico específico en que el impacto se produce, derivado de la interconexión de los mercados en un mundo globalizado.

En este contexto, la uE publicó en el año 2007 el Libro Verde sobre adaptación al Cambio Climático para la Unión Europea, en el cual reconoce el importante rol que le cabe en la materia (Dannevig, Rauken y Hovelsrud, 2012), estableciendo cuatro pilares dirigidos a las siguientes áreas (CCE, 2007): 1) acciones inmediatas en la Unión Europea, 2) integración de la adaptación a las acciones externas de la $\mathrm{UE}^{17}, 3$ ) perfeccionamiento del conocimiento, y 4) fomento de la participación de todas las partes interesadas.

De estos cuatro pilares, el primero resulta de especial interés para los países miembros de la UE ya que promueve la integración y el

\footnotetext{
16 A través de la implementación del Sistema Europeo de Comercio de Derecho de Emisión. Ver http://europa. eu/legislation_summaries/other/128109_es.htm

17 Al respecto véase la Comunicación de la Comisión al Consejo y al Parlamento Europeo: El cambio climático en el contexto de la cooperación al desarrollo. сом (2003) 85 final.
} 
desarrollo normativo de la adaptación, estableciendo además la típica división según sectores (por ejemplo, agricultura, infraestructura, salud, etc.); división que, como se verá, fue semejante a la utilizada en el Programa Nacional de Adaptación del Reino Unido ${ }^{18}$.

Por otro lado, mientras el Libro Verde contiene posiciones generales con el objetivo de promover la reflexión y discusión al interior de los Estados miembros frente a una problemática determinada, el Libro Blanco contiene propuestas concretas de acción comunitaria para abordar las cuestiones planteadas por el Libro Verde. Por ello, conviene detenerse en el Libro Blanco para observar cuál fue la aproximación de la Unión Europea respecto a la forma de enfrentar la adaptación.

El Libro Blanco tuvo como objetivo establecer un marco "para reducir la vulnerabilidad de la ue al impacto del cambio climático", el cual fue concebido como un complemento a las medidas adoptadas por cada Estado (CCE, 2009). Asimismo, a pesar de la mirada global del problema, el marco comunitario que propuso el Libro Blanco tuvo, al igual que su predecesor, el Libro Verde, un enfoque regionalista (o localista) y sectorial ${ }^{19}$.
En cuanto a la estructura, el Libro Blanco se basa en cuatro pilares de acción: 1) generar conocimientos sobre impactos y consecuencias del cambio climático para la UE; 2) integrar la adaptación en las políticas clave de la UE; 3) utilizar una combinación de instrumentos estratégicos (instrumentos de mercado, asociaciones público-privadas, etc.) para garantizar la eficacia de la adaptación; y 4) reforzar la cooperación internacional en la materia (CCE, 2009).

De esta manera, en relación con la generación de conocimientos, se anuncia la promoción de un mecanismo de intercambio de información sobre impactos, vulnerabilidad y mejores prácticas en materia de adaptación ${ }^{20}$. A través de esta se apuntó a recolectar conocimientos sobre los impactos del fenómeno climático y sus consecuencias en el territorio comunitario, para luego comenzar un lento, pero progresivo, proceso de integración de la adaptación a las políticas de la Unión Europea (Dannevig et al., 2012).

La integración de la adaptación a las políticas comunitarias (segundo pilar) tiene una doble expresión: por una parte, promueve la reorientación o modificación de "cada una de las áreas políticas para facilitar la adaptación”,

18 La comunicación señala: "es necesario prever una acción inmediata, integrando en la normativa y en algunas políticas la cuestión de la adaptación al cambio climático. Esta integración ha de atender a aquellos sectores que se ven o se verán fuertemente afectados por el cambio climático, como la agricultura y la silvicultura, los transportes, la salud pública, el agua, la pesca, los ecosistemas y la biodiversidad, y debe abordar cuestiones transversales como las evaluaciones de impacto ambiental y la protección civil” (CCE, 2007).

19 Cabe destacar la diferencia semántica entre "aumentar la resistencia”, utilizada en este documento, y la expresión "resiliencia" utilizada hoy en día para referirse al aumento de la adaptación.

20 Lo que se tradujo posteriormente en la aplicación web denominada European Climate Adaptation Platform (Climate-adapt13), o Plataforma Europea de Adaptación al Clima (cE, 2013). 
lo que se traduce en un proceso de revisión de diversas normativas e instrumentos de política comunitaria $^{21}$; y por la otra, se reconoce la libertad que tienen los Estados para regular respecto de ciertos sectores económicos, por ejemplo, infraestructura física. Finalmente, se señala que la Comisión

...va a trabajar con los Estados miembros y las partes interesadas en el establecimiento de orientaciones y el intercambio de buenas prácticas para garantizar que se tengan en cuenta los impactos del cambio climático al aplicar la Directiva de Evaluación de Impacto Ambiental (EIA) y la Directiva de Evaluación Ambiental Estratégica (EAE), así como las políticas de ordenación territorial (CCE, 2009).

El último instrumento formulado al alero de la UE en esta materia es la Estrategia de Adaptación, publicada el año 2013. Dicho documento señala como objetivo: "contribuir a una Europa más resistente al clima. Ello supone mejorar la preparación y la capacidad de respuesta a los efectos del cambio climático a nivel local, regional, nacional y de la UE, creando un planteamiento coherente y mejorando la coordinación" (cE, 2013). Para la consecución del objetivo propuesto se establecen tres ejes: 1) el fomento de la actuación de los Estados miembros a través del desarrollo de estrategias nacionales de adaptación, con planteamientos comunes y coherentes entre sí; 2) la generación y el mejoramiento de in- formación y 3) la promoción de la adaptación en los sectores vulnerables.

La influencia de la política de adaptación de la UE en el ámbito doméstico queda de manifiesto en el Programa de Adaptación del Reino Unido, el cual declara, al referirse a la Estrategia de Adaptación de la UE, que:

...los Estados miembros no están legalmente obligados a satisfacer estas directrices, ya que las mismas están diseñadas para ayudar a los países de la UE a desarrollar, implementar y revisar las políticas de adaptación de acuerdo con sus circunstancias nacionales. Sin embargo, el gobierno espera que el NAP esté ampliamente en línea con las futuras directrices (HM Government, 2013b),

Subrayando de paso que dicha estrategia "se enfoca en ayudar a llenar los vacíos de evidencia y fortalecer el reparto de conocimiento, datos y mejores prácticas entre los Estados miembros, a través del Climate-ADAPT" (нM Government, 2013b). Se advierte así una retroalimentación permanente entre la política de adaptación desarrollada en el orden interno de cada país miembro - en este caso Reino Unido- y aquella promovida en el ámbito supranacional por la Unión Europea.

Finalmente, cabe subrayar que la labor de adaptación desplegada por la UE rebasa el marco de la política del cambio climático, manifestándose a través de otras iniciativas particulares, por ejemplo, en la implementa-

21 Por ejemplo, el Programa Sanitario y la Estrategia Sanitaria de la UE, la Directiva sobre Inundaciones, la Directiva Marco sobre el Agua, la Estrategia para la Escasez del Agua y las Sequías, la Directiva Marco sobre la Estrategia Marina, etc. 
ción del Marco de Acción de Hyogo del año $2005^{22}$, lo cual ha sido reconocido por la UE en la comunicación del año 2014 denominada: "Marco de Acción de Hyogo, posterior a 2015: gestionar los riesgos para lograr la resiliencia", en la cual se destacan "las sinergias sólidas con la adaptación al cambio climático”.

En síntesis, es posible constatar que la influencia de la UE en relación con la adaptación al cambio climático de los Estados miembros se ejerce por dos vías: la primera, a través del desarrollo de una política comunitaria de adaptación, que en poco más de un lustro ha generado un Libro Verde, un Libro Blanco y una Estrategia de Adaptación; y la segunda, mediante la promoción de la adaptación al cambio climático por parte de los países miembros, en el desarrollo de otras políticas de interés comunitario, como es el caso de la implementación del marco de Hyogo. En este contexto, resulta notoria la semejanza en la evolución de la política de adaptación en el ámbito comunitario y en el de la Convención.

\section{LA POLÍTICA DE ADAPTACIÓN AL CAMBIO CLIMÁTICO EN EL REINO UNIDO}

La política de cambio climático del Reino Unido es reconocida a nivel mundial ${ }^{23}$, tanto en mitigació, como en adaptación. El éxito alcanzado por este país es atribuible en gran medida al marco legal establecido por la Climate Change Act del año 2008, aunque también ha sido significativo el aporte realizado por diferentes organismos que, con anterioridad a la promulgación y entrada en vigencia de este cuerpo legal, han colaborado en la satisfacción de los objetivos de la CMNUCC.

El mérito de la Climate Change Act es que instaura normativamente una institucionalidad particular para la adaptación, a través de dos instrumentos: el Programa Nacional de Adaptación (NAP) y la Evaluación de Riesgos del Cambio Climático (CCRA, por sus siglas en inglés). Asimismo, crea un órgano especializado en adaptación, el Subcomité de Adaptación, y faculta al ministro del Department of Environment Food and Rural Affairs (DEFRA) para requerir la elaboración de informes de adaptación de parte de ciertas entidades públicas y privadas, facultad

22 El Marco de Acción de Hyogo tiene tres objetivos estratégicos: 1) la integración más efectiva de la consideración de los riesgos de desastre en las políticas, los planes y los programas de desarrollo sostenible a todo nivel, con acento especial en la prevención y mitigación de los desastres, la preparación para casos de desastre y la reducción de la vulnerabilidad; 2) la creación y el fortalecimiento de instituciones, mecanismos y medios a todo nivel, en particular a nivel de la comunidad, que puedan contribuir de manera sistemática a aumentar la resiliencia ante las amenazas, y 3) en la fase de reconstrucción de las comunidades damnificadas, la incorporación sistemática de criterios de reducción de riesgos en el diseño y la ejecución de los programas de preparación para las situaciones de emergencia, de respuesta y de recuperación (Naciones Unidas, 2005).

23 Reino Unido es uno de los países ocDE más avanzados en términos de investigación y progreso en el diseño e implementación de políticas de adaptación (Mullan, Kingsmill, Kramer y Agrawala, 2013). 
denominada Adaptation Reporting Power (ARP). A continuación se revisarán cada uno de estos elementos.

1. El Programa Nacional de Adaptación. Es el instrumento de política pública más importante en materia de adaptación ${ }^{24}$. En este se establecen los objetivos, las medidas y los plazos en relación con la adaptación al cambio climático para Reino Unido. El primero de estos programas, publicado en julio de 2013, tuvo como objetivos generales: 1) incrementar la conciencia (ante la adaptación); 2) aumentar la resiliencia ante eventos extremos; 3) tomar acciones oportunas para la ejecución de medidas; y 4) enfrentar los vacíos más importantes. A estos se añaden dos problemáticas transversales que permean todo el NAP: inundaciones y presión sobre los recursos hídricos (HM Government, 2013b).

Las medidas para la satisfacción de estos objetivos se agrupan en los siguientes sectores: construcción de ambientes, infraestructura, salud y comunidades resilientes, agricultura y silvicultura, ambientes naturales, negocios y gobiernos locales. En cada una hay "una visión particular de lo que es necesario asegurar para que el sector esté adecuadamente preparado" y una o más áreas de interés (focus área) que relevan aquellos aspectos que serán abordados. De esta manera, cada uno de los capítulos que compone el NAP comienza citando el o los riesgos específicos que han sido identificados en el CCRA, planteando a continuación objetivos precisos y una o más acciones, para la satisfacción de tales objetivos ${ }^{25}$. Cabe señalar que el NAP carece de exigibilidad, es decir, no hay una norma específica que haga obligatoria la ejecución de las medidas que contiene. Además, dado que los destinatarios del NAP pueden ser entidades públicas o privadas, las medidas solo serán obligatorias, si estas forman parte de la función del organismo (por ejemplo, reducción de inundaciones en las agencias del sector de infraestructura); mientras que para el sector privado no habrá por regla general modo de obligar a la ejecución de las acciones del NAP ${ }^{26}$.

24 Sección 56 (1), (3) y 58 (United Kingdom, 2008).

25 Robert Hitchin, funcionario del Asc, en entrevista personal, señala que una vez que los riesgos han sido identificados, se forman equipos por sectores del NAP (por ejemplo salud, en el cual él participa), los cuales luego desarrollan las medidas de acuerdo con un diálogo con distintos actores involucrados en el sector correspondiente. Mientras tanto, DEFRA promueve la realización de debates, talleres, etc., destinados a facilitar este diálogo.

26 La implementación efectiva de las medidas es diferente tratándose de empresas reguladas y el sector privado en general. Para las primeras, los requerimientos establecidos por la ley les obligan a cumplir con las medidas de adaptación, por ejemplo, la continuidad del suministro energético. En cuanto a las segundas, si bien las medidas no son exigibles, su ejecución reporta beneficio a los destinatarios y, por tanto, tendrán incentivos para el cumplimiento. 
Como una forma de reforzar la implementación efectiva de las medidas se establecieron facilitadores en todo el proceso. Por ejemplo, la selección de las acciones en la etapa de formulación del Nap fue realizada en conjunto con sus futuros destinatarios, a través de la conformación de equipos interinstitucionales y la asesoría de un organismo especializado, el Domestic Adaptation Board ${ }^{27}$, lo cual contribuyó al involucramiento del sector público. Mientras tanto, los reportes bajo el ARP (según veremos más adelante) y la realización de talleres de trabajo y debates favorecieron la participación de la sociedad civil. Por su parte, en la etapa de implementación el Gobierno presta asesoría en la ejecución de las medidas a través del Climate Ready Support Service $^{28}$, un servicio que orienta a los usuarios sobre cómo adaptarse incorporando administración de riesgos climáticos en sus decisiones de negocios (HM Government, 2013b) y la Environment Agency ${ }^{29}$.

Desde ya es posible advertir la correlación entre este Programa de Adaptación $\mathrm{y}$ los lineamientos provenientes desde el régimen internacional en al menos cinco aspectos. En primer lugar, el segundo objetivo del NAP no es sino una reiteración del primero de los objetivos a que deben ceñirse los planes nacionales de adaptación, según las recomendaciones del Marco de Adaptación de Cancún. En efecto, aumentar la resiliencia permite reducir la vulnerabilidad y, de ese modo, contribuir a la adaptación al cambio climático.

En segundo lugar, el NAP está orientado a la implementación de acciones concretas que en general apuntan al perfeccionamiento de las estrategias de reducción de riesgos de desastres relacionados con el cambio climático, lo cual fue impulsado también por el Marco de Adaptación de Cancún.

En tercer lugar, el NAP recoge las recomendaciones tanto del Marco de Cancún, como del Libro Verde de la ue (Pilar Tercero), en la delimitación de roles de los sectores privado y público. Como resultado de esta reflexión se sostuvo que al sector privado le corresponde hacerse cargo de sus propios riesgos, mientras que el Estado le asiste un deber de asesoramiento en dicho proceso. Esta lógica, se afirma, permitió que el sector privado

\footnotetext{
27 "El Domestic Adaptation Board, es un comité compuesto por funcionarios senior provenientes de diferentes sectores del Gobierno, en el cual se promueve la integración de la adaptación en las demás políticas claves del Gobierno de modo de generar una respuesta común frente a dicha problemática" (DECC, 2013) (Mullan et al., 2013).

28 Ver http://test.environment-agency.gov.uk/cy/ymchwil/137557.aspx

29 A través de dicho servicio, la Environment Agency complementa los roles y las responsabilidades que le corresponden sobre riesgos de inundación, erosión costera, protección de recursos hídricos y protección de ambientes acuáticos y humedales (DECC, 2013).
} 
se involucrara y gestionara sus riesgos de manera anticipada y responsable ${ }^{30}$. En cuarto lugar, y como una cuestión terminológica, es de notar que el NAP es un programa y no un plan; dado que, como se vio más arriba, los programas de adaptación son anteriores a los planes, estaban principalmente dirigidos a los países menos adelantados y perseguían la captación de financiamiento para la ejecución de las medidas en ellos contenidas. Sin embargo, la comparación de los objetivos generales del NAP del Reino Unido y los objetivos propuestos para los planes de adaptación bajo el Marco de Cancún demuestra que el primero sigue de cerca las recomendaciones señaladas por esta COP.

Finalmente, y desde una perspectiva formal, el NAP hace eco de la ordenación por sectores propuesta por el Libro Verde de la Unión Europea.

2. Evaluación de Riesgos del Cambio Climático. Contiene en un solo documento la mejor información disponible relativa a los riesgos del cambio climático, siendo el insumo base para las decisiones por plasmar en el NAP correspondiente ${ }^{31}$. Así por ejemplo, en el CCRA que sirvió de base para el NAP 2013 se identificaron alrededor de setecientos riesgos potenciales, profundizándose luego en el análisis de cien de ellos, priorizados según urgencia, magnitud de las consecuencias previstas y costo-efectividad asociado ${ }^{32}$. La ley prescribe que estos reportes se realizarán cada cinco ańos, publicándose el primero en enero de 2012.

Este instrumento de la Climate Change Act releva la insoslayable relación entre el conocimiento científico y la formulación de la política pública, lo cual ha estado presente tanto al interior del régimen internacional del cambio climático, mediante los informes del PICC, como en el ámbito de la Unión Europea, en relación al Pilar Tercero del Libro Verde.

3. Subcomité de Adaptación. En tercer lugar, la ley estableció un organismo de carácter independiente conformado por profesionales y académicos de reconocida experiencia, el Subcomité de Adaptación (ASC, por sus siglas en inglés), cuya función consiste en proporcionar consejo experto, análisis e información en ${ }^{33}: 1$ ) la preparación del CCRA; 2) la implemen-

\footnotetext{
30 Según informaciones recibidas en entrevista personal por Robert Hitchin.

31 "En la selección de las áreas por priorizar, nos guiamos por las calificación de magnitud, seguridad y urgencia asignadas durante el análisis que sustenta el CCRA. El foco se puso en los riesgos que requieren atención urgente debido a la expectativa segura de impactos de alta magnitud y aquellos con largos horizontes de planificación, por ejemplo, proyectos de gran infraestructura” (нм Government, 2013b).

32 Aunque el CCRA debe ser elaborado por DEFRA, el primero fue encargado a una entidad privada - Wallinford LTD.- que obtuvo la licitación de dicho proyecto (Webb, 2011). En entrevista personal realizada a Robert Hitchin, se aclara que el próximo CCRA se está desarrollando al interior de DEFRA.

33 Schedule 1. The Committee on Climate Change, párrafo 16 (10) (United Kingdom, 2008).
} 
tación del Programa de Adaptación para Inglaterra; y 3) la asesoría en adaptación a las autoridades nacionales de Gales, Escocia y el Norte de Irlanda ${ }^{34}$. Este organismo, administrativamente no depende de DEFrA, sino que es parte del Comité de Cambio Climático (CCC), organismo ad hoc creado por la Climate Change $\mathrm{Act}^{35}$. Con ello se asegura la objetividad necesaria para escrutar la aplicación del NAP por parte de DEFRA y los demás órganos comprometidos, evaluación que posteriormente debe ser remitida al Parlamento, señalando las principales fortalezas y debilidades identificadas ${ }^{36}$.

En el proceso de elaboración del primer CCRA se puede observar que la intervención del ASC superó las exigencias establecidas por la ley, en pos de la con- secución de su mandato, interviniendo con antelación a los plazos establecidos en la ley de cambio climático ${ }^{37}$, a efectos de presentar al Parlamento un CCRA consensuado entre DEFRA (y sus licitantes) y el $\mathrm{ASC}^{38}$.

La creación de una entidad independiente, con el mandato de monitorear la ejecución del NAP es otro aspecto que se ajusta a las directrices emanadas desde el ámbito internacional, en tanto estas enfatizan que "la planificación de la adaptación es un proceso continuo, progresivo e iterativo".

4. El Adaptation Reporting Power. Finalmente, la Climate Change Act faculta al ministro de DEFRA para requerir a las autoridades públicas ${ }^{39} \mathrm{y}$ a ciertas empresas definidas en la ley (statutory undertaker ${ }^{40}$

34 El Reino Unido se compone de Inglaterra, Gales, Escocia e Irlanda del Norte; estas tres últimas son delegaciones que poseen autonomía regulatoria respecto de ciertas materias, entre la cuales se encuentra el cambio climático. De ahí que si bien la gestión es centralizada, la planificación para la adaptación queda en manos de cada Autoridad Delegada. De esta manera, Escocia publicó en 2009 la Scotland's Climate Change Adaptation Framework, mientras que Gales publicó la Climate Change Strategy for Wales and Associated Emission Reduction and Adaptation Delivery Plans en 2010, e Irlanda del Norte el Northern Ireland Climate Change Adaptation Programme (DECC, 2013).

35 Sección 56 (5), 57 (1) (United Kingdom, 2008).

36 En este sentido, la ley ordena que el Asc, a través del Comité de Cambio Climático, deberá preparar evaluaciones del progreso realizado hacia la consecución de los objetivos y las acciones establecidos en el NAP, con el propósito de identificar y trabajar aquellos aspectos en que se adviertan debilidades o vacíos de información (Commons, 2015).

37 Esto es, con seis meses de anterioridad a la fecha final establecida para la presentación del CCRA ante el Parlamento. Sección 57 (2) (United Kingdom, 2008).

38 Adicionalmente, el Asc colaboró en la evaluación económica de la adaptación, lo cual, aunque no estaba mandatado expresamente por la ley, demuestra la proactividad de dicho órgano como consecuencia de las amplias atribuciones conferidas para el desarrollo de su cometido (Least Developed Countries Expert Group, 2012).

39 Sección 70 (1) (United Kingdom, 2008).

40 La sección 98 (6) de la Environmental Protection Act 1990 establece un catálogo definido de cuáles serán consideradas "Statutory undertaker". Entre estas empresas se encuentran los operadores de trenes, líneas férreas y sistemas ferroviarios urbanos, los transportistas terrestres, fluviales y marítimos y los operadores de puertos y aeropuertos (United Kingdom, 1990). 
y empresas de servicios públicos) informes sobre la evaluación de los impactos del cambio climático dentro de sus respectivas ámbitos, las acciones que tomarán para hacerles frente y los plazos. En ejercicio de esta atribución el ministro de DEFRA puede establecer requerimientos especiales, tales como reportes conjuntos, plazos específicos, áreas geográficas determinadas, etc. ${ }^{41}$. El ejercicio de esta facultad está orientado a generar tres consecuencias positivas: instalar y promover la reflexión sobre la problemática de la adaptación en el sector; permitir a la autoridad conocer el estado de preparación para la adaptación a un nivel de profundidad que difícilmente se podría obtener desde fuera de cada entidad en particular, y finalmente, consensuar medidas de adaptación específicas entre la autoridad y el sector privado ${ }^{42}$.

Hasta aquí la descripción de las principales instituciones creadas por la Climate Change Act en relación con la adaptación. Ahora bien, este diseño institucional ha sido objeto de críticas, por ejemplo, la falta de imparcialidad de que adolecería el NAP en tanto este reflejaría la priorización de DEFRA de los riesgos identificados en el CCRA, o también la posibilidad de que dichos instrumentos materialicen una aproximación vertical o top-down a la problemática de la adaptación. En efecto, si los límites de la evidencia base son determinados por la autoridad, la respuesta institucional resultará sesgada respecto a los focos de atención que han sido relevados, afectando la selección de los riesgos priorizados y la participación de la sociedad civil al interior del proceso ${ }^{43}$.

Una última crítica tiene que ver con las facultades otorgadas al AsC para la evaluación de la implementación del NAP. Lo anterior en la medida en que la definición de las metodologías para la evaluación están en manos del Gobierno de turno (Hм Government, 2013b), con lo cual es posible desvirtuar la eficacia de esta importante herramienta de monitoreo en la ejecución de la política de adaptación.

Desde otra perspectiva, adjudicar los progresos del Reino Unido en materia de adaptación exclusivamente a la existencia de la Climate Change Act omitiría la labor realizada durante al menos una década anterior

41 Sección 61 a 63 (United Kingdom, 2008).

42 Para una revisión de la implementación de esta facultad véase Hм Government (2013a).

43 Los defensores de esta configuración contraargumentan que la labor del Gobierno consiste precisamente en administrar intereses, privilegiando unos por sobre otros y que, en último término, la evidencia científica es uno entre otros argumentos, tales como los factores sociales o económicos involucrados en la decisión gubernamental. Para este tema no existe una única respuesta, sin embargo, lo que no puede perderse de vista es la relevancia de la evidencia científica como parte del fundamento legitimante en la toma de decisiones (Boyd, 2013). 
a la entrada en vigencia de dicha ley ${ }^{44}$. En este sentido, la OCDE ha destacado que las primeras medidas de adaptación de parte del Gobierno del Reino Unido comenzaron en la década de los noventa con la creación del Programa de Impactos Climáticos del Reino Unido (UKCIP), destinado a crear mayores vínculos entre la comunidad científica y los responsables de las políticas ${ }^{45}$.

En este sentido, la revisión de diferentes documentos e instrumentos de política que sirvieron de antecedente para la elaboración del NAP confirman que la adaptación fue desarrollada incluso con anterioridad a la Climate Change Act, en el marco de la política del desarrollo sustentable ${ }^{46}$. En estos se señala, por ejemplo, que el diseño de la política de adaptación debe considerar tres criterios: efectividad, eficiencia y equidad. Mientras los dos primeros persiguen reducir la vulnerabilidad de manera efectiva y a bajo costo, el tercero guarda relación con la evaluación de los aspectos distributivos de las medidas, ponderando los impactos que su aplicación tendrá dentro de cada sector de la sociedad en el corto y largo plazo. Este último, considera también un elemento intergeneracional que analiza los impactos de las decisiones presentes sobre las acciones futuras, es decir, la sustentabilidad involucrada en el diseño de las medidas (Cimato y Mullan, 2010).

De manera más evidente el Informe de la Comisión Parlamentaria del año 2010, recomienda al DEFRA reevaluar las estimaciones de los beneficios de largo plazo de la adaptación para mejorar la valoración del medio ambiente en la evaluación de políticas ${ }^{47}$.

44 Por ejemplo, a principios de 2008 el Gobierno inglés publicó "Adapting to climate change in England: A framework for Action" (Defra, 2008).

45 Lo mismo ha sido reiterado en la Sexta Comunicación Nacional de dicho país. "El Programa de uk sobre cambios climáticos fue establecido en 1997 para aconsejar a los stakeholder-led sobre evaluación de cambio climático. [...] Las proyecciones del ukcP09 producidas por la Met Office Hadley Centre han entregado información sobre una escala espacial e incluye una evaluación sobre el grado de certeza en los resultados. Estas proyecciones fueron usadas en el primer UK CCRA" (DECC, 2013; Mullan et al., 2013).

46 El concepto del desarrollo sustentable en el Reino Unido tiene cada vez mayor consagración legal, lo cual ha sido explicado como consecuencia de su instalación como principio de política pública no solo en dicho país, sino también a nivel comunitario. Lo anterior no obsta para que, muchas veces, la concepción del desarrollo sustentable no sea uniforme, pudiendo adoptar el carácter de objetivo (es decir, como un propósito general de la regulación), o bien como un deber de contenido específico. En cualquier caso, la capacidad de aplicación depende en gran medida de las diferentes redacciones. Se afirma, no obstante, que esta diversidad contribuye a enriquecer el concepto y a instalar un debate sobre su contenido siempre dinámico y de comprobada eficacia a esta urgente y necesaria disciplina de la política pública (Ross, 2008).

47 En el sistema inglés, los Comités Parlamentarios fiscalizan la actividad del Gobierno. En ejecución de esta labor, pueden examinar el gasto, la administración y las políticas, pudiendo requerir información a las diferentes entidades involucradas. Como resultado se elabora un reporte con las audiencias realizadas, las recomendaciones del Parlamento y las respectivas respuestas del Gobierno. En este contexto, el Environmental Audit Committe "evalúa en qué medida las políticas y los programas de los ministerios y de los órganos públicos no ministeriales contribuyen a la protección ambiental y al desarrollo sustentable” (Commons, 2010). 
Adicionalmente, cada Ministerio y sus respectivas agencias ejecutivas debió elaborar planes de acción de desarrollo sustentable (SDAP, por sus siglas en inglés), en los cuales intervino el Programa de Adaptación al Cambio Climático a efectos de incorporar medidas de adaptación dentro de la evaluación (Commons, 2010). Desde una perspectiva presupuestaria, el marco del desarrollo sustentable también es evaluado mediante reportes financieros anuales que los ministerios deben dirigir al Tesoro. Esta última entidad posee la facultad para determinar requerimientos específicos entre los cuales se encuentra la adaptación al cambio climático (Commons, 2013).

Por último, la propia Climate Change Act requiere que los objetivos y las políticas establecidos en el NAP contribuyan al desarrollo sustentable ${ }^{48}$, factor que entre otras cosas incide en los roles que en dicho documento se asignan al Gobierno y a la sociedad civil.

De esta manera, la gobernanza de la adaptación al cambio climático en el Reino Unido se inscribe en el proceso de consolidación del desarrollo sustentable, existiendo un diálogo institucional permanente entre las agencias involucradas en uno y otro ámbito, lo cual responde a las directrices formuladas a propósito de los programas y los planes nacionales de adaptación, y a la propia Convención, cuando expresa que para el logro de los objetivos del artículo segundo se debe considerar el desarrollo sustentable ${ }^{49}$.

Por último, de las relaciones anteriormente expresadas ha surgido el concepto de adaptación sustentable, el cual ha sido definido al interior del proceso deliberativo de la adaptación en uK como un compañero esencial del desarrollo sustentable, "que apunta a asegurar que las medidas de adaptación no contribuyan a las causas o consecuencias del cambio climático, y que las acciones en un sector o lugar no limiten de manera irrazonable la capacidad de otras acciones para adaptarse de manera exitosa" (Commons, 2010).

\section{CONCLUSIONES}

El objetivo de este artículo fue presentar la política de adaptación al cambio climático del Reino Unido, en relación con las directrices emanadas del ordenamiento jurídico internacional, las cuales, al no ser vinculantes, respetan la libertad de los Estados para decidir la fórmula más adecuada de acuerdo con sus circunstancias nacionales.

A través de este ejercicio se ha podido concluir que existe una sincronía permanente

\footnotetext{
48 Sección 58 (2), Parte 4, "The objectives, proposals and policies must be such as to contribute to sustainable development” (United Kingdom, 2008).

49 Artículo 3(4): "Las Partes tienen derecho al desarrollo sostenible y deberían promoverlo. Las políticas y medidas para proteger el sistema climático contra el cambio inducido por el ser humano deberían ser apropiadas para las condiciones específicas de cada una de las Partes y estar integradas en los programas nacionales de desarrollo, tomando en cuenta que el crecimiento económico es esencial para la adopción de medidas encaminadas a hacer frente al cambio climático" (Naciones Unidas, 1992).
} 
entre el orden internacional y el ámbito doméstico que se expresa en la creación de una arquitectura institucional y la elaboración de instrumentos, programas y planes, los cuales se insertan en el resto del engranaje jurídico político, prestando particular atención a la política del desarrollo sustentable.

El ejemplo del Reino Unido, si bien ha sido calificado por sus propios gestores como un primer paso $^{50}$, es notable por iniciar a través de una ley, un proceso institucional destinado a generar una política pública de adaptación al cambio climático de largo plazo, iterativa e independiente de los intereses y las motivaciones del Gobierno de turno. El mérito de la ley, en consecuencia, consiste en poner en marcha un proceso autorreflexivo, movilizando los diferentes interesados hacia la consecución de un objetivo común.

Por otro lado, es necesario subrayar que aunque la ley prescribe la elaboración de un programa de adaptación, ello no implica que el contenido de dicho instrumento de política pública sea posteriormente exigible. Sin embargo, es posible apreciar que esta suerte de precariedad normativa no obsta para que, en la práctica, sea ejecutado por sus destinatarios, sean estos públicos o privados.

Por otra parte, la política de adaptación abre un espacio de reflexión de carácter participativo sobre la medida en que el Estado debe involucrarse y hacerse cargo de una problemática en que confluyen aspectos de carácter público, pero también privado, legitimando la actividad estatal y contribuyendo al involucramiento y participación de los demás agentes en dicho proceso.

A modo de cierre, conviene recordar que Colombia se ha propuesto, en su Plan Nacional de Desarrollo 2014-2018, la creación de una ley de cambio climático, presentándola como un objetivo en la Contribución Prevista Determinada a Nivel Nacional (INDC, por sus siglas en inglés) enviada a la Secretaría de la Convención (Colombia, 2015). Este argumento por sí solo debería dirigir nuestra atención hacia los aprendizajes que puedan extraerse de la experiencia comparada.

\section{REFERENCIAS}

Bauer, A., Feichtinger, J. Steurer, R. (2012). The Governance of Climate Change Adaptation in 10 OECD Countries: Challenges and Approaches. Journal of Environmental Policy y Planning, 14 (3), 279-304.

Biagini, B., Bierbaum, R., Stults, M., Dobardzic, S. y McNeeley, S. M. (2014). A typology of adaptation actions: A global look at climate adaptation actions financed through the Global Environment Facility. Global Environmental Change, 25 (1), 97-108.

Biesbroek, G. R., Swart, R. J., Carter, T. R., Cowan, C., Henrichs, T., Mela, H. et al. (2010). Europe adapts to climate change: Comparing National Adaptation Strategies. Global Environmental Change, 20 (3), 440-450. Recuperado de http:// doi.org/10.1016/j.gloenvcha.2010.03.005

50 Entrevista a lord Krebs, jefe del Asc (Keenan y Krebs, 2014). 
Boyd, I. (2013). Making science count in government. eLife (2), 2-5.

CCE (2007). Libro Verde de adaptación al cambio climático en Europa: opciones de actuación para la UE (vol. сом-2007). Recuperado de http://eurlex.europa.eu/LexUriServ/site/es/com/2007/ com2007_0354es01.pdf

CCE (2009). Comisión de las Comunidades Europeas.

Libro Blanco. Adaptación al cambio climático: hacia un marco europeo de actuación. Recuperado de http://eur-lex.europa.eu/LexUriServ/ LexUriServ.do?uri=COM:2009:0147:FIN:ES:PDF CE (2013). Estrategia de adaptación al cambio climático de la Unión Europea.

Cimato, F. y Mullan, M. (2010). Adapting to Climate Change: Analysing the Role of Government. London.

CMNUCC (1995). Informe de la Conferencia de las Partes sobre su primer periodo de sesiones, celebrado en Berlín del 28 de marzo al 7 de abril de 1995 (vol. 61658, pp. 1-57). Recuperado de http://unfccc.int/resource/docs/spanish/cop1/ g9561658.pdf\#page $=4$

CMNUCC (1998). Informe de la Conferencia de las Partes sobre su cuarto periodo de sesiones, celebrado en Buenos Aires del 2 al 14 de noviembre de 1998 (vol. 60439). Recuperado de http://unfccc.int/resource/docs/spanish/cop4/cp416a01s. pdf\#page $=4$

CMNUCC (2001). Informe de la Conferencia de las Partes sobre su séptimo periodo de sesiones, celebrado en Marrakech del 29 de octubre al 10 de noviembre de 2001 (vol. 60246, p. 40). Recuperado de http://unfccc.int/resource/docs/2015/cop21/ spa/109s.pdf

CMNUCC (2010). Informe de la Conferencia de las Partes sobre su $16^{\circ}$ periodo de sesiones, celebrado en Cancún del 29 de noviembre al 10 de diciembre de 2010 (vol. 60553, pp. 1-33). Recuperado de http://unfccc.int/resource/docs/2010/cop16/ spa/07a01s.pdf\#page $=2$

CMNUCC (2011). Informe de la Conferencia de las Partes sobre su 17 periodo de sesiones, celebrado en Durban del 28 de noviembre al 11 de diciembre de 2011 (vol. 60551, pp. 1-24). Recuperado de http://unfccc.int/resource/docs/2011/cop17/ spa/09a01s.pdf

CMNUCC. (2012). Informe de la Conferencia de las Partes sobre su $18^{\circ}$ periodo de sesiones, celebrado en Doha del 26 de noviembre al 8 de diciembre de 2012 (vol. 60411, p. 40). Recuperado de http://unfccc.int/resource/docs/2015/cop21/ spa/109s.pdf

CMNUCC (2014). Informe de la Conferencia de las Partes sobre su $20^{\circ}$ periodo de sesiones, celebrado en Lima del 1 al 14 de diciembre de 2014 (vol. 01804, p. 40). Recuperado de http://unfccc.int/ resource/docs/2015/cop21/spa/109s.pdf

Colombia (2015). Contribución Prevista Determinada a Nivel Nacional (iNDC). Recuperado de http:// www4.unfccc.int/submissions/Indc/Published Documents/Colombia/1/INDC Colombia.pdf

Commons, H. of. (2010). Adapting to climate change. Sixth Report of Session 2009-10. TR News. Recuperado de papers2://publication/uuid/BD0D4D30-96A4-47F4-9EAFF5896ADB6C47

Commons, H. of. (2013). Embedding sustainable development: An update. First Report of Session 2013-14. Recuperado de http://www.publications.parliament.uk/pa/cm201314/cmselect/ cmenvaud/202/202.pdf

Commons, H. of. (2015). Change, Committee on Climate Annual Report and Accounts 14/15. Recuperado de https://www.theccc.org.uk/wp-content/ uploads/2015/06/2903902_CCC_ARA_acc.pdf

OPERA, N¹9.Julio-Diciembre 2016.pp. 11-34 
Dannevig, H., Rauken, T. y Hovelsrud, G. (2012). Implementing adaptation to climate change at the local level. Local Environment, 17 (6-7), 597-611.

DECC (2013). The UK's Sixth National Communication and First Biennial Report under the UNFCCC. London. Defra (2008). Adapting to climate change in England. Policy Paper, 1-52.

Econoticias.com. (2011) Alcanzar un acuerdo vinculante en Durban es un "cuento de hadas". Recuperado de http:/www.ecoticias.com/co2/42362/noticiasmedio-ambiente-medioambiente-medioambiental-ambiental-definicion-contaminacion-cambio-climatico-calentamiento-global-ecologiaecosistema-impacto-politica-gestion-legislacioneducacion-responsabilidad-tecnico-sos

Ejecucción, Ó. S. de. (2013). Informe de la $23^{\circ}$ reunión del Grupo de Expertos para los paises menos adelantados (vol. 61658).

HM Government (2013a). Adapting to Climate Change: Ensuring Progress in Key Sectors 2013 Strategy for exercising the Adaptation Reporting Power and list of priority reporting authorities.

HM Government. (2013b). The National Adaptation Programme: Making the Country Resilient to a Changing Climate.

Huq, S. y Toulmin, C. (2006). Three eras of climate change. Sustainable Delevolpment Opinion. Recuperado de http://pubs.iied.org/pdfs/11060IIED. pdf

Keenan, C. E. T. R. y Krebs, L. (2014). In Conversation with Lord Krebs: full transcript. Recuperado dehttps://theconversation.com/in-conversationwith-lord-krebs-full-transcript-24575

Least Developed Countries Expert Group (2012). National Adaptation Plans: Technical Guidelines for the National Adaptation Plan Process. Recuperado de http://unfccc.int/files/adaptation/ cancun_adaptation_framework/application/pdf/ naptechguidelines_eng_high__res.pdf

Mehling, M. (2009). El cambio climático y la Unión Europea entre ambición y realidad. En Remiro Brotons, A. y Fernández Egea, R. M. (eds.). El cambio climático en el derecho internacional y comunitario. Bilbao: Fundación BBVA. Recuperado dehttp://cataleg.upc.edu/ record $=\mathrm{b} 1350592 \sim \mathrm{S} 1^{*}$ cat

Mullan, M., Kingsmill, N., Kramer, A. M. y Agrawala, S. (2013). National Adaptation Planning: Lessons from $O E C D$ Countries. Recuperado de http:// dx.doi.org/10.1787/5k483jpfpsq1-en

Naciones Unidas (1992). Convención marco de las Naciones Unidas sobre el cambio climático, 62301 Convención Marco de las Naciones Unidas sobre el Cambio Climático 98. Recuperado de http:// scholar.google.com/scholar?hl=en \&btnG $=$ Sea rch\&q=intitle:Convenci? $\mathrm{n}+$ marco+de+las+na ciones+unidas+sobre+el+cambio+clim?tico\#1

Naciones Unidas (2005). Informe de la Conferencia Mundial sobre la Reducción de los Desastres (vol. 72, p. 975). Ginebra: onU.

Reid, C. (2012). Climate Law in the United Kingdom. En Hollo, K. M. M., Kulovesi, E. (ed.). Climate Change and the Law (pp. 537-549). USA: Springer.

Ross, A. (2008). Why legislate for sustainable development? An examination of sustainable development provisions in UK and scottish statutes. Journal of Environmental Law, 20 (1), 35-68.

Schipper, E. L. F. (2006). Conceptual history of adaptation in the UNFCCC process. Review of European Community and International Environmental Law, 15 (1), 82-92.

Schlosberg, D. (2013). Political Challenges of the Climate-Changed Society. PS: Political Science y Politics, 46 (01), 13-17. 
unFCCC (2006). Manual Convención Marco de las Naciones Unidas sobre el Cambio Climático. Bonn: ONU.

United Kingdom (1990). Environmental Protection Act, Environmental Protection Act. Recuperado de http://www.legislation.gov.uk/ukpga/1990/43/ pdfs/ukpga_19900043_en.pdf

United Kingdom (2008). Climate Change Act. Recuperado de http://doi.org/10.1136/ bmj.39469.569815.47

Urrutia, O. (2010). El régimen jurídico internacional del cambio climático después del "Acuerdo de Copenhague”. Revista de Derecho, 34, 597-634.

Verschuuren, J. (2012). Legal Aspects of Climate Change Adaptation. En Hollo, K.-M. M. y Kulovesi,
E. (eds.). Climate Change and the Law (pp. 257285). USA: Springer.

Vihma, A. (2012). Analyzing soft Law and Hard Law in Climate Change. En Hollo, K. M. M. y Kulovesi, E. (eds.). Climate Change and the Law (pp. 143.164). USA: Springer.

Viñuales, J. E. (2009). El régimen jurídico internacional relativo al cambio climático: perspectivas y prospectivas. Recuperado de http://www.oas.org/dil/ esp/9 - viñuales.233-306.pdf

Webb, J. (2011). Making climate change governable: the case of the UK climate change risk assessment and adaptation planning. Science and Public Policy, 38 (4), 279-292. 